\title{
Scleral Fixation of a Hydrophobic Acrylic Intraocular Lens with Eyelets Using 8-0 Polypropylene Suture
}

\author{
Do Wook Kim¹, Sung Chul Lee ${ }^{2}$, Ji Hwan Lee ${ }^{1}$ \\ ${ }^{1}$ Department of Ophthalmology, The Institute of Vision Research, Yonsei University College of Medicine, Seoul, Korea \\ ${ }^{2}$ Department of Ophthalmology, Konyang University College of Medicine, Daejeon, Korea
}

Purpose: To report clinical outcomes of a scleral fixation technique of a hydrophobic acrylic intraocular lens with eyelets using 8-0 polypropylene suture.

Methods: Nine eyes of nine patients who underwent combined pars plana vitrectomy and sclera fixation of an intraocular lens using this technique were analyzed.

Results: The mean follow-up period was 7.11 months (range, 6-12 months), and there was a significant visual improvement at 6 months after surgery. The mean logarithm of the minimum angle of the resolution changed from 0.54 at baseline to 0.29 at postoperative 6 months $(p=0.016)$. The mean postoperative spherical equivalent at 6 months was $-0.90 \pm 0.90$ diopters, and the mean predictive error was $-0.49 \pm 0.62$ diopters.

Conclusions: Postoperative visual and refractive outcomes were favorable, and the positions of intraocular lenses were well centered in all cases. This technique could be a useful alternative for surgeons without easy access to Gore-Tex suture.

Key Words: Intraocular lenses, Polypropylenes, Sclera, Surgery

Secondary intraocular lens (IOL) implantation is often necessary in the absence of zonular support after successful cataract extraction. Various surgical techniques have been introduced including anterior chamber IOL, iris-sutured IOL, scleral-sutured IOL, sutureless intrascleral fixation, and iris-claw IOL [1-8]. The selection of surgical technique usually depends on each patient's ocular condition and surgeon preference. The advantage of scleral-sutured fixation over other techniques is placing the IOL in the ana-

Received: August 2, 2021 Final revision: November 2, 2021

Accepted: November 5, 2021

Corresponding Author: Ji Hwan Lee, MD. Department of Ophthalmology, Yongin Severance Hospital, Yonsei University College of Medicine, 363 Dongbaekjukjeondae-ro, Giheung-gu, Yongin 16995, Korea. Tel: 8231-5189-8495, Fax: 82-31-5189-8566, E-mail: leejihwan@yuhs.ac tomic position away from anterior chamber structures.

Recently, favorable outcomes have been demonstrated using Gore-Tex suture (W. L. Gore \& Associates, Newark, DE, USA) for IOL fixation to the sclera [9-11]. Although there are multiple advantages in using Gore-Tex suture including its long-term durability and positive outcomes, it is difficult to get the material in some countries because of importing issues. Therefore, we report the result of this scleral fixation technique using 8-0 polypropylene suture instead of Gore-Tex suture.

\section{Materials and Methods}

This scleral fixation technique was performed in nine eyes of nine patients between April 1, 2019 and 
October 31, 2019. All patients underwent combined pars plana vitrectomy (PPV) and scleral fixation of an IOL, and all surgeries were performed by a single surgeon (JHL). This retrospective, interventional case series followed the tenets of the Declaration of Helsinki and was was approved by the institutional review board of Severance Hospital, Yonsei University College of Medicine (No. 9-2020-0167). Informed consent was waived due to the retrospective nature of this study.

Conjunctival peritomy with a relaxing incision was performed at the temporal and nasal side. The horizontal meridians were marked using ASICO axis marker (Westmont, IL, USA) at 3 and 9 o'clock positions of the limbus. An infusion cannula was inserted at the inferotemporal side, $3 \mathrm{~mm}$ away from the limbus. Four sclerotomy sites were marked $3 \mathrm{~mm}$ away from the limbus and $4 \mathrm{~mm}$ apart from each other.
Two valved trocars were placed at the two superior marks. A standard PPV with scleral depression and shaving of vitreous base was performed. A clear corneal incision was made at the superior limbus using a 2.75-mm keratome. Two strands of 8-0 polypropylene sutures (Ethicon, Somerville, NJ, USA) with the needle removed were used to form two loops on each side. The suture was passed through each eyelet of a MX60 IOL (Bausch and Lomb, Bridgewater, NJ, USA) with both ends of the suture going over the haptic. Each end of the suture was placed into the anterior chamber and positioned at the pupillary area behind the iris plane. The suture was retrieved through the corresponding sclerotomy site using intraocular forceps. A similar pattern of suture passage was repeated with the other suture through the opposite sclerotomy sites. The IOL optic was folded in half using a Kelman-Mcpherson forcep, and the IOL was
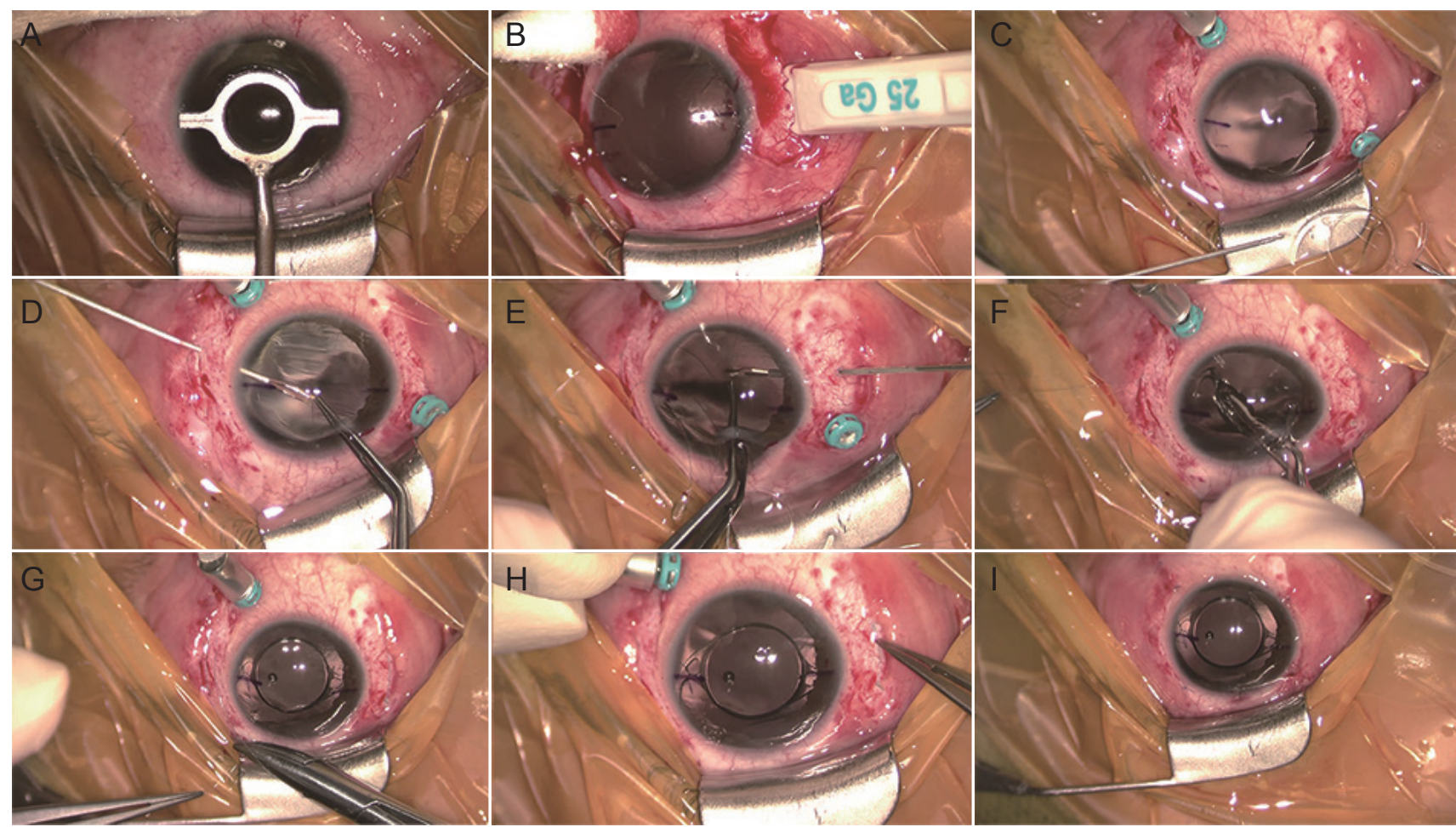

Fig. 1. Intraoperative findings of scleral fixation of a hydrophobic acrylic intraocular lens with eyelets using 8-0 polypropylene suture. (A) The horizontal meridians were marked at 3 and 9 o'clock positions at the limbus. (B) Four sclerotomy sites were marked 3 mm from the limbus and $4 \mathrm{~mm}$ apart. Pars plana vitrectomy was performed through a sclerotomy site. (C) An 8-0 polypropylene suture was passed through each eyelet of the intraocular lens with both ends of the suture going over the haptic. (D) Each end of the suture was placed into the anterior chamber and retrieved through the corresponding sclerotomy using intraocular forceps. (E) A similar pattern of suture passage was repeated with the other suture through the opposite sclerotomy. (F) The intraocular lens optic was folded in half and inserted into the anterior chamber. $(\mathrm{G})$ The sutures were tied using a 3-1-1 technique. (H) The knots were trimmed and buried into the sclerotomy site. (I) The corneal incision and conjunctival peritomies were sutured. 


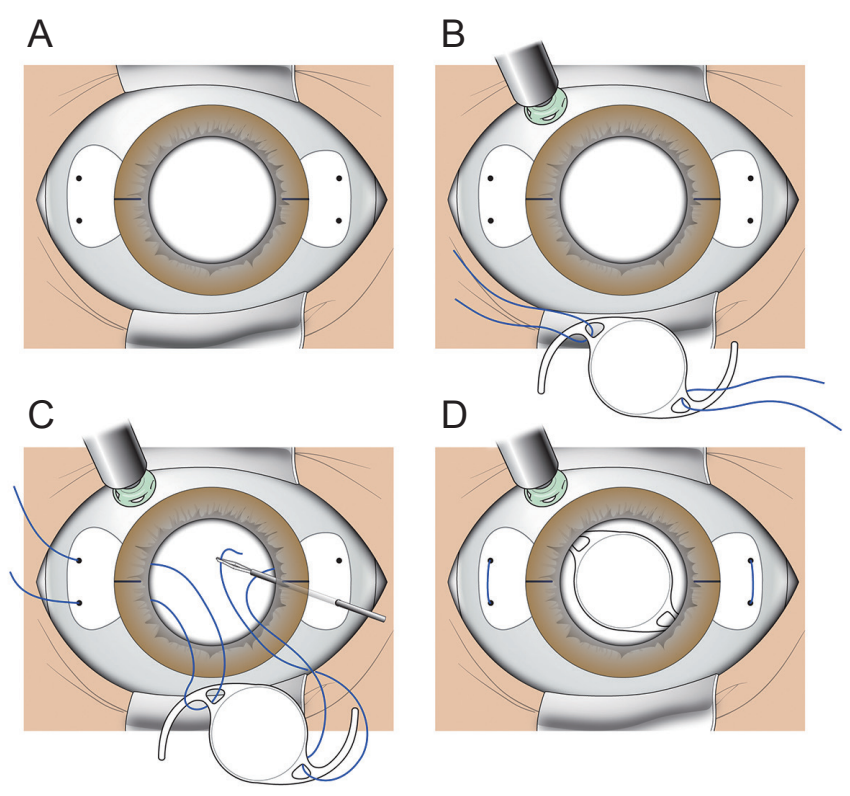

Fig. 2. Schematic drawing of scleral fixation of a hydrophobic acrylic intraocular lens with eyelets using 8-0 polypropylene suture (surgeon's view, superior position). (A) After conjunctival peritomy was performed at the temporal and nasal side, four sclerotomy sites were marked $3 \mathrm{~mm}$ from the limbus and $4 \mathrm{~mm}$ apart. (B) An 8-0 poylpropylene suture was passed through each eyelet of the intraocular lens with both ends of the suture going over the haptic. (C) Each end of the suture was placed into the anterior chamber and retrieved through the corresponding sclerotomy using intraocular forceps. (D) After inserting the intraocular lens, the sutures were tied and buried into the sclerotomy site.

introduced in the anterior chamber. After confirming appropriate suture tension and centration of the IOL, the sutures were tied using a 3-1-1 technique. The knots were trimmed and buried into the sclerotomy site. The corneal incision and conjunctival peritomies were sutured (Fig. 1A-1I, 2A-2D).

Preoperative lens calculations were performed using optical biometry (IOLMaster; Carl Zeiss Meditec, Dublin, CA, USA). A routine in-the-bag calculation was used because sclerotomies were made $3 \mathrm{~mm}$ posterior to the limbus. The formula used in each IOL calculation was based on axial length. For axial length less than $22.5 \mathrm{~mm}$, the Hoffer Q formula was used. The Sanders-Retzlaff-Kraff theoretical formula was used for axial lengths over $22.5 \mathrm{~mm}$. Postoperative refractive error was measured at 6 months after surgery. Postoperative lens astigmatism was calculated using the method previously described by Munoz-Escriva and Furlan [12], $\left[\begin{array}{ll}\mathrm{S}+\mathrm{C} \sin ^{2} \alpha & -\mathrm{C} \sin \alpha \cos \alpha \\ -\mathrm{C} \sin \alpha \cos \alpha & \mathrm{S}+\mathrm{C} \sin ^{2} \alpha\end{array}\right]$ in the standard notation spherical (S) / cylinder $(\mathrm{C}) \times$ axis $(\alpha)$ [13].

\section{Results}

Baseline characteristics and clinical outcomes are summarized in Table 1 . The mean age was 57.56 years and the mean follow-up period was 7.11 months (range, 6-12 months). Indications for surgery included dislocated IOL (five eyes), postoperative aphakia (three eyes), and subluxated crystalline lens (one eye). There was a significant visual improvement at 6 months after surgery. The mean logarithm of the minimum angle of the resolution change from 0.54 at baseline to 0.29 at postoperative 6 months ( $p=0.016$ ). The mean postoperative spherical equivalent (SE) at 6 months was $-0.86 \pm 0.82$ diopters (D), and the mean predictive error (postoperative SE minus target refraction) was $-0.49 \pm 0.62 \mathrm{D}$. The Sanders-Retzlaff-Kraff theoretical formula was used in eight patients and the Hoffer Q formula was used in one patient with axial length $22.36 \mathrm{~mm}$. Prediction error in this patient was $-0.05 \mathrm{D}$, which was smaller than the mean prediction error of patients with Sanders-Retzlaff-Kraff theoretical formula $(-0.58 \pm 0.64$ D). The IOLs were well centered in all cases without any tilt, subluxation, or dislocation. The mean calculated lens astigmatism at postoperative 6 months was $1.31 \pm 0.49 \mathrm{D}$, which was similar to that of conventional two-point fixation technique by the same surgeon $(1.04 \pm 0.89 \mathrm{D}, p=0.394, \mathrm{n}=7)$. No intraoperative complication was encountered. Postoperative complications included transient corneal edema in two eyes $(22.2 \%)$, cystoid macular edema in two eyes (22.2\%), and increased intraocular pressure controlled with medical therapy in one eye $(11.1 \%)$. There was no case of postoperative hypotony, endophthalmitis, suture breakage, IOL dislocation, or retinal detachment during the follow-up period.

\section{Discussion}

The selection of suture material is crucial to a longterm postoperative prognosis in cases of IOL scleral 
Table 1. Baseline characteristics and clinical outcomes of patients $(n=9)$

\begin{tabular}{lc}
\hline Characteristics & Value \\
\hline Age (yr) & $57.56 \pm 15.70$ \\
Sex (male : female) & $7: 2$ \\
Duration of follow-up (mon) & $7.11 \pm 1.96$ \\
Indications for surgery & $5(55.6)$ \\
Dislocated intraocular lens & $3(33.3)$ \\
Postoperative aphakia & $1(11.1)$ \\
Crystalline lens subluxation & $0.54 \pm 0.63$ \\
Best-corrected visual acuity (logMAR) & $0.29 \pm 0.33$ \\
Preoperative & 0.016 \\
Postoperative 6 month & $-0.86 \pm 0.82$ \\
p-value & $-0.49 \pm 0.62$ \\
Spherical equivalent at postoperative 6 month (D) & $1.31 \pm 0.49$ \\
Prediction error (D) & $2(22.2)$ \\
Calculated lens astigmatism at postoperative 6 month (D) & $2(2.2)$ \\
Postoperative complications & $11.1)$ \\
Corneal edema &
\end{tabular}

Values are presented as mean \pm standard deviation or number (\%).

$\log \mathrm{MAR}=$ logarithm of the minimum angle of resolution; $\mathrm{D}=$ diopters.

fixation. The use of 10-0 polypropylene suture is becoming less popular because of the suture breakage resulting in IOL subluxation or dislocation, which have been reported to be as high as $24 \%$ after 10 years [14]. In 2014, Kahn et al. [9] described a technique for scleral fixation of IOL using Gore-Tex suture. Subsequent short-term and 1-year follow-up outcomes demonstrated the effectiveness and safety of a trans-scleral IOL fixation using Gore-Tex suture $[10,11]$. Recently, a study comparing this technique with combined PPV with anterior chamber IOL revealed similar visual outcomes with reduced postoperative complications including early corneal edema [15]. Despite its high tensile strength and resistance to degradation, it is difficult to use Gore-Tex suture in some countries including South Korea because it is not commercially imported locally. In addition, Gore-Tex suture is not yet indicated for ophthalmologic use in the manufacturer's label, and further studies regarding its long-term safety results are necessary. John et al. [16] recently described a technique of scler- al IOL fixation using 8-0 polypropylene and Akreos AO60 IOL (Bausch and Lomb) instead of 10-0 polypropylene or Gore-Tex suture, which showed good clinical outcomes with safety results.

Our technique demonstrated favorable visual and refractive outcomes with acceptable short-term complications. Postoperative prediction error $(-0.49 \pm 0.62$ D) showed slightly myopic outcomes than targeted. Although transscleral fixation is known to have a higher refractive prediction error than other techniques of secondary IOL implantation [17], the refractive outcomes in scleral fixation tend to vary from myopic [18,19] to hyperopic differences [20]. Recently, sutureless scleral fixation of IOL has been described, of which refractive outcomes were also varied. Whereas Todorich et al. [21] reported the mean final refraction as $-0.565 \mathrm{D}$, Abbey et al. [22] revealed that the prediction error was +0.41 D. Combined PPV might be a possible factor accounting for the myopic shift [23], but the exact mechanism is still unknown.

Our technique has the following advantages: first, a 
hydrophobic acrylic IOL was used to avoid the risk of lens opacification associated with gas or oil tamponade as reported previously [24]; second, 8-0 polypropylene suture has enough stiffness that the suture can stay stable in the pupillary area, allowing this technique to be also performed without needing to use the handshake maneuver; and third, we demonstrated acceptable refractive outcomes when suturing $3 \mathrm{~mm}$ behind the limbus (postoperative SE, -0.86 at 6 months; prediction error, $-0.49 \mathrm{D}$ ). According to Su et al. [25], the mean postoperative SE and the mean prediction error for fixation $3 \mathrm{~mm}$ posterior to the limbus were -0.82 and $-0.43 \mathrm{D}$, respectively. Compared with sclerotomies placed $2 \mathrm{~mm}$ posterior to the limbus, creating sclerotomies $3 \mathrm{~mm}$ behind the limbus can result in less myopic shift and minimize iris chafing or vitreous hemorrhage.

There are several important points to be highlighted in this technique. First, consistency in sclerotomy site placement is crucial to prevent unpredictable refractive results. Every sclerotomy site should be measured and marked carefully to confirm the exact distance from the limbus and from each other. A slight anterior placement in sclerotomy may result in a myopic shift and vice versa. Second, suture tension is another important factor. If the suture is too tight, the lens may tilt and be decentered. If the suture is too loose, the lens may tremble along with eyeball movement, leading to unpredictable refractive outcomes. Third, careful preoperative assessment of the conjunctiva is crucial for preventing postoperative suture exposure. The position of sclerotomy site should be made with consideration to previous surgical interventions on the conjunctiva such as glaucoma surgery. Lastly, smaller gauge instruments would be advantageous for preventing postoperative leakage from the sclerotomy site.

This report has several limitations, including its retrospective nature and small number of cases. Visual and refractive outcomes may be influenced by preoperative condition of the eye because various surgical indications and complicated preoperative histories were present. In addition, 23 or $25 \mathrm{G}$ vitrectomy systems were used in this case series, which could have affected the refractive outcomes. However, we believe that these results revealed an evidence-based outcome in performing combined vitrectomy and scleral fixation of a hydrophobic acrylic IOL using 8-0 polypropylene suture. Further long-term outcome results with a larger number of cases would be necessary to confirm the surgical outcomes of this technique.

In summary, we report clinical outcomes of a scleral fixation technique of a hydrophobic acrylic IOL with eyelets using 8-0 polypropylene suture. Postoperative visual and refractive outcomes were favorable, and the position of IOLs were well centered in all cases. This technique could be a useful alternative for surgeons without easy access to Gore-Tex suture.

\section{Conflicts of Interest}

No potential conflict of interest relevant to this article was reported.

\section{Acknowledgements}

The authors thank MID (Medical Illustration \& Design) for providing excellent support with medical illustration.

\section{Funding}

The authors received no financial support for this article.

\section{References}

1. Bellucci R, Pucci V, Morselli S, Bonomi L. Secondary implantation of angle-supported anterior chamber and scleral-fixated posterior chamber intraocular lenses. J Cataract Refract Surg 1996;22:247-52.

2. Donaldson KE, Gorscak JJ, Budenz DL, et al. Anterior chamber and sutured posterior chamber intraocular lenses in eyes with poor capsular support. J Cataract Refract Surg 2005;31:903-9.

3. Vote BJ, Tranos P, Bunce C, et al. Long-term outcome of combined pars plana vitrectomy and scleral fixated sutured posterior chamber intraocular lens implantation. Am J Ophthalmol 2006;141:308-12.

4. Baykara M, Ozcetin H, Yilmaz S, Timucin OB. Posterior iris 
fixation of the iris-claw intraocular lens implantation through a scleral tunnel incision. Am J Ophthalmol 2007;144:586-91.

5. Gabor SG, Pavlidis MM. Sutureless intrascleral posterior chamber intraocular lens fixation. J Cataract Refract Surg 2007;33:1851-4.

6. Kim SJ, Lee SJ, Park CH, et al. Long-term stability and visual outcomes of a single-piece, foldable, acrylic intraocular lens for scleral fixation. Retina 2009;29:91-7.

7. De Silva SR, Arun K, Anandan M, et al. Iris-claw intraocular lenses to correct aphakia in the absence of capsule support. J Cataract Refract Surg 2011;37:1667-72.

8. Yamane S, Sato S, Maruyama-Inoue M, Kadonosono K. Flanged intrascleral intraocular lens fixation with double-needle technique. Ophthalmology 2017;124:1136-42.

9. Khan MA, Gerstenblith AT, Dollin ML, et al. Scleral fixation of posterior chamber intraocular lenses using gore-tex suture with concurrent 23-gauge pars plana vitrectomy. Retina 2014;34:1477-80.

10. Khan MA, Gupta OP, Smith RG, et al. Scleral fixation of intraocular lenses using Gore-Tex suture: clinical outcomes and safety profile. Br J Ophthalmol 2016;100:638-43.

11. Khan MA, Samara WA, Gerstenblith AT, et al. Combined pars plana vitrectomy and scleral fixation of an intraocular lens using Gore-Tex suture: one-year outcomes. Retina 2018;38:1377-84.

12. Munoz-Escriva L, Furlan WD. Statistical analysis when dealing with astigmatism: assessment of different spherocylindrical notations. Ophthalmic Epidemiol 2001;8:27-37.

13. Shin JY, Choi SR, Jeon JH, et al. Temporary haptic externalization and four-point fixation of intraocular lens in scleral fixation to enhance stability. Korean J Ophthalmol 2018;32:23-8.

14. Asadi R, Kheirkhah A. Long-term results of scleral fixation of posterior chamber intraocular lenses in children. Ophthalmology 2008;115:67-72.

15. Khan MA, Gupta OP, Pendi K, et al. Pars plana vitrectomy with anterior chamber versus Gore-Tex sutured posterior chamber intraocular lens placement: long-term outcomes. Retina 2019;39:860-6.

16. John T, Tighe S, Hashem O, Sheha H. New use of 8-0 polypropylene suture for four-point scleral fixation of secondary intraocular lenses. J Cataract Refract Surg 2018;44:1421-5.

17. Brunin G, Sajjad A, Kim EJ, et al. Secondary intraocular lens implantation: complication rates, visual acuity, and refractive outcomes. J Cataract Refract Surg 2017;43:369-76.

18. Hayashi K, Hayashi H, Nakao F, Hayashi F. Intraocular lens tilt and decentration, anterior chamber depth, and refractive error after trans-scleral suture fixation surgery. Ophthalmology 1999;106:878-82.

19. Lockington D, Ali NQ, Al-Taie R, et al. Outcomes of scleral-sutured conventional and aniridia intraocular lens implantation performed in a university hospital setting. $J$ Cataract Refract Surg 2014;40:609-17.

20. Yang JM, Yoon KC, Ji YS. Transscleral fixation of single-piece foldable acrylic lens with eyelets at the optic-haptic junction. Can J Ophthalmol 2015;50:367-72.

21. Todorich B, Stem MS, Kooragayala K, et al. Structural analysis and comprehensive surgical outcomes of the sutureless intrascleral fixation of secondary intraocular lenses in human eyes. Retina 2018;38 Suppl 1(Suppl 1):S31-40.

22. Abbey AM, Hussain RM, Shah AR, et al. Sutureless scleral fixation of intraocular lenses: outcomes of two approaches. The 2014 Yasuo Tano Memorial Lecture. Graefes Arch Clin Exp Ophthalmol 2015;253:1-5.

23. Hamoudi H, La Cour M. Refractive changes after vitrectomy and phacovitrectomy for macular hole and epiretinal membrane. J Cataract Refract Surg 2013;39:942-7.

24. Kalevar A, Dollin M, Gupta RR. Opacification of scleral-sutured Akreos AO60 intraocular lens after vitrectomy with gas tamponade: case series. Retin Cases Brief Rep 2020;14:174-7.

25. Su D, Stephens JD, Obeid A, et al. Refractive outcomes after pars plana vitrectomy and scleral fixated intraocular lens with Gore-Tex suture. Ophthalmol Retina 2019;3:54852. 